\title{
Association between preoperative activity level and functional outcome at 12 months following surgical decompression for lumbar spinal stenosis
}

\author{
Galal Elsayed, MD, ${ }^{1}$ Matthew S. Erwood, MD, ${ }^{1}$ Matthew C. Davis, MD, MPH, ${ }^{1}$ \\ Esther C. Dupépé, MD, ${ }^{1}$ Samuel G. McClugage III, MD, 1 Paul Szerlip, PhD, ${ }^{2}$ \\ Beverly C. Walters, MD, MSc, FRCSC, ${ }^{1}$ and Mark N. Hadley, MD ${ }^{1}$ \\ 'Department of Neurosurgery, University of Alabama at Birmingham, Alabama; and 'Department of Computer Science, University
} of Central Florida, Orlando, Florida

\begin{abstract}
OBJECTIVE This study defines the association of preoperative physical activity level with functional outcomes at 3 and 12 months following surgical decompression for lumbar spinal stenosis.

METHODS Data were collected as a prospective observational registry at a single institution from 2012 through 2015, and then analyzed with a retrospective cohort design. Patients who were able to participate in activities outside the home preoperatively were compared to patients who did not participate in such activities, with respect to 3-month and 12-month functional outcomes postintervention, adjusted for relevant confounders.

RESULTS Ninety-nine patients were included. At baseline, sedentary/inactive patients $(n=55)$ reported greater back pain, lower quality of life, and higher disability than similarly treated patients who were active preoperatively. Both cohorts experienced significant improvement from baseline in back pain, leg pain, disability, and quality of life at both 3 and 12 months after lumbar decompression surgery. At 3 months postintervention, sedentary/inactive patients reported more leg pain and worse disability than patients who performed activities outside the home preoperatively. However, at 12 months postintervention, there were no statistically significant differences between the two cohorts in back pain, leg pain, quality of life, or disability. Multivariate analysis revealed that sedentary/inactive patients had improved disability and higher quality of life after surgery compared to baseline. Active patients experienced greater overall improvement in disability compared to inactive patients.
\end{abstract}

CONCLUSIONS Sedentary/inactive patients have a more protracted recovery after lumbar decompression surgery for spinal stenosis, but at 12 months postintervention can expect to reach similar long-term outcomes as patients who are active/perform activities outside the home preoperatively.

https://thejns.org/doi/abs/10.3171/2018.2.SPINE171028

KEYWORDS physical activity; neurosurgery; lumbar stenosis; outcome assessment; adult

工 UMBAR spinal stenosis is a common symptomatic surgically treatable pathology for which an estimated annually, a 4-fold increase since $1985^{2,6,19}$ Variations in surgical practice, increased access to spine imaging, and an aging and increasingly obese population are cited for the rising rates of lumbar decompression procedures in the United States and Europe., ${ }^{71}$
Surgical decompression for lumbar spinal stenosis demonstrates mixed long-term outcomes. ${ }^{13}$ Successful outcomes have been published in ranges from $26 \%$ to $100 \%$ of patients. ${ }^{33}$ Some investigators cite significant improvements in overall pain and disability reported after decompression in elderly patients. ${ }^{21}$ Others have described short-term outcomes of up to $80 \%$ improvement, but with far less durable long-term outcomes. Equipoise in the out-

ABBREVIATIONS EQ-5D = EuroQol-5 Dimensions health survey; ODI = Oswestry Disability Index; $U A B=$ University of Alabama at Birmingham. SUBMITTED September 15, 2017. ACCEPTED February 14, 2018.

INCLUDE WHEN CITING Published online July 6, 2018; DOI: 10.3171/2018.2.SPINE171028. 
comes of conservatively and surgically managed patients with lumbar spinal stenosis have been reported at 4 to 5 years postoperatively. . $^{13,20,28}$

A recent trend has been to use prospectively collected clinical registries to evaluate the quality of lumbar spinal stenosis care and to optimize healthcare resource distribution. ${ }^{4,8} \mathrm{~A}$ key field of investigation involves identification of preoperative factors and/or patient characteristics that may affect patient-reported outcomes after surgical intervention for lumbar spinal stenosis. . $^{13,26,33}$

One proposed factor in the outcome of lumbar spinal stenosis after decompression is the level of the patient's preoperative activity. ${ }^{1}$ To our knowledge, no studies have described the impact of activity outside the home with respect to postoperative outcomes for lumbar spinal stenosis. The purpose of this study was to determine how the degree of participation in activities outside the home is associated with patient-reported outcomes at 3 and 12 months after surgical decompression for symptomatic lumbar spinal stenosis.

\section{Methods \\ Data Collection}

Data were collected in a prospective observational registry of patients treated for symptomatic lumbar spinal stenosis, and who were enrolled between February 2012 and June 2015. The database included morbidity, mortality, and patient-reported outcomes at baseline, 3 months, and 12 months postintervention. The methodology for collection, data abstraction, and implementation has been previously described. 5,6,23 Data coordinators at the University of Alabama at Birmingham (UAB) entered the data through a secure portal (https://projectredcap.org). Data were exported from the secure portal in a de-identified format. The UAB Institutional Review Board considers the collection and analysis of these data exempt from its review due to a previous assessment of the methodologies of the data collection. ${ }^{34}$

\section{Study Design}

This study was designed as a retrospective observational cohort study of prospectively collected data that aims to characterize the association between patient activity outside the home and functional patient-reported outcomes after decompression surgery for lumbar spinal stenosis. Patients were stratified by self-reported activity outside the home at their preoperative baseline. The aims were to determine functional status of each patient cohort at baseline and postoperatively, and to determine the value of preoperative functional activity as a prognostic indicator for postoperative functional outcomes. The STROBE (Strengthening the Reporting of Observational Studies in Epidemiology) guidelines were followed for reporting of observational studies. ${ }^{35}$

\section{Study Measures}

The following patient-reported functional metrics were assessed at baseline, at 3 months postintervention, and at 12 months postintervention: back pain and leg pain over the prior 7 days were measured on a 0 - to 10 -point visual analog scale, EuroQol-5 Dimensions (EQ-5D) health survey, ${ }^{10}$ and Oswestry Disability Index (ODI). ${ }^{11}$

\section{Statistical Analysis}

Univariate descriptive statistics of the two patient cohorts were assessed. Parametric data were compared via the Student t-test, and related parametric data sets were compared using paired t-tests. Normality was assessed using the Shapiro-Wilk test. Nominal and ordinal data were compared using a Fisher exact test. An alpha level of 0.05 was considered significant. All statistical analyses were performed using IBM SPSS software (version 23.0).

Multivariate analysis was performed with multiple regression models with continuous dependent variables and categorical independent variables. Analyses were performed for baseline data, 12-month data, and the margin of difference between baseline and 1 year after intervention. The covariates included age stratified by decade, sex, smoking status, diabetes, reported depression, BMI, and ambulation type. These covariates are derived as confounders from the SPORT (Spine Patient Outcomes Research Trial) lumbar stenosis prospective analyses..$^{5}$ The multiple regression analyses were performed using RStudio (version 0.99.902) and $\mathrm{R}$ programming language (version 3.2.3).

\section{Results}

\section{Demographics and Baseline Clinical Factors}

Of the 99 patients included in this study, $76 \%$ have 12-month follow-up data. The patients are divided into two cohorts: strenuous to moderate activity outside the home $(n=44)$ or sedentary/light to no activity outside the home $(\mathrm{n}=55)$. The analysis of baseline characteristics (Table 1) demonstrates that patients who did not participate in activities outside the home have higher rates of daily smoking, non-insulin-dependent type 2 diabetes mellitus, and a higher American Society of Anesthesiologists (ASA) grade. A total of 18 patients underwent minimally invasive operations, 6 in the active cohort and 12 in the sedentary/light activity group. Complications for the two cohorts have been included as well, with a slightly higher rate in the sedentary one (Table 2). The sedentary/inactive cohort reported greater back pain, more disability, and worse quality of life preoperatively compared to patients in the active cohort (Table 3 ).

\section{Postoperative Patient-Reported Outcomes}

At 3 months postintervention, sedentary patients without activity outside the home continued to report greater leg pain and more disability after surgery than the active cohort. By 12 months postintervention, however, there were no significant differences between the two cohorts in back pain, leg pain, quality of life, or disability (Table 3 ).

\section{Functional Outcomes Over Time}

Seventy-six patients were eligible for paired testing due to completeness of data points between baseline, 3 months, and 12 months postintervention. Relative to their respective baselines, both the group of patients who were active outside the home $(n=33)$ and the group who did 
TABLE 1. Baseline characteristics of cohorts

\begin{tabular}{|c|c|c|c|}
\hline Characteristic, $\mathrm{N}=99$ & Activities Outside the Home, $n=44$ & No Activities Outside the Home, $n=55$ & p Value \\
\hline Mean age in yrs, \pm SD & $63.71 \pm 13.65$ & $59.79 \pm 12.89$ & 0.151 \\
\hline Mean BMI score, \pm SD & $27.52 \pm 5.53$ & $29.87 \pm 7.02$ & 0.076 \\
\hline \multicolumn{4}{|l|}{ Sex } \\
\hline Male & $32(72.73)$ & $31(56.36)$ & 0.14 \\
\hline Female & $12(27.27)$ & $24(43.64)$ & 0.14 \\
\hline \multicolumn{4}{|l|}{ Insurance payer } \\
\hline Medicare & $23(52.27)$ & $23(41.82)$ & 0.405 \\
\hline Medicaid & $0(0.00)$ & $0(0.00)$ & 1 \\
\hline Private & $20(45.45)$ & $24(43.64)$ & 1 \\
\hline Veterans Affairs/government & $1(2.27)$ & $7(12.73)$ & 0.119 \\
\hline Uninsured & $0(0.00)$ & $1(1.82)$ & 1 \\
\hline \multicolumn{4}{|l|}{ Smoker } \\
\hline Currently every day or almost daily & $3(6.82)$ & $15(27.27)$ & 0.015 \\
\hline Currently some days or occasionally & $0(0.00)$ & $1(1.82)$ & 1 \\
\hline Do not know if currently smoking & $0(0.00)$ & $0(0.00)$ & 1 \\
\hline Not a current smoker & $41(93.18)$ & 39 (70.91) & 0.009 \\
\hline \multicolumn{4}{|l|}{ Diabetes } \\
\hline Type 2, non-insulin-dependent & $1(2.27)$ & 10 (18.18) & 0.022 \\
\hline Type 2, insulin-dependent & $1(2.27)$ & $4(7.27)$ & 0.517 \\
\hline Type 1, insulin-dependent & $0(0.00)$ & $0(0.00)$ & 1 \\
\hline No & $42(95.45)$ & $41(74.55)$ & 0.008 \\
\hline \multicolumn{4}{|l|}{ Depression disorder } \\
\hline Yes & $5(11.36)$ & $12(21.82)$ & 0.269 \\
\hline No & $39(88.64)$ & $43(78.18)$ & 0.269 \\
\hline \multicolumn{4}{|l|}{ Level of education } \\
\hline High school diploma or GED & $11(25.00)$ & $13(23.64)$ & 1 \\
\hline Less than high school & $0(0.00)$ & $3(5.45)$ & 0.335 \\
\hline 2-yr college degree & $5(11.36)$ & $11(20.00)$ & 0.378 \\
\hline 4-yr college degree & $14(31.82)$ & $15(27.27)$ & 0.784 \\
\hline Post-college & $14(31.82)$ & $13(23.64)$ & 0.495 \\
\hline \multicolumn{4}{|c|}{ Patient participation in activities outside the home? } \\
\hline Strenuous & $10(22.73)$ & $0(0.00)$ & $<0.001$ \\
\hline Moderate & $34(77.27)$ & $0(0.00)$ & $<0.001$ \\
\hline Sedentary or light & $0(0.00)$ & $20(36.36)$ & $<0.001$ \\
\hline None & $0(0.00)$ & $35(63.64)$ & $<0.001$ \\
\hline \multicolumn{4}{|c|}{ Patient participation in activities inside the home? } \\
\hline Strenuous & $3(6.82)$ & $2(3.64)$ & 0.789 \\
\hline Moderate & $28(63.64)$ & $8(14.55)$ & $<0.001$ \\
\hline Sedentary or light & 8 (18.18) & $30(54.55)$ & $<0.001$ \\
\hline None & $5(11.36)$ & $15(27.27)$ & 0.084 \\
\hline \multicolumn{4}{|l|}{ ASA grade } \\
\hline 1 & $2(4.55)$ & $1(1.82)$ & 0.832 \\
\hline 2 & $16(36.36)$ & $9(16.36)$ & 0.041 \\
\hline 3 & $26(59.09)$ & $44(80.00)$ & 0.041 \\
\hline 4 & $0(0.00)$ & $1(1.82)$ & 1 \\
\hline \multicolumn{4}{|l|}{ No. of levels decompressed } \\
\hline 1 & $17(38.64)$ & $14(25.45)$ & 0.235 \\
\hline 2 & $16(36.36)$ & $26(47.27)$ & 0.376 \\
\hline 3 & $6(13.64)$ & $12(21.82)$ & 0.434 \\
\hline 4 & $3(6.82)$ & $3(5.45)$ & 1 \\
\hline
\end{tabular}


» CONTINUED FROM PAGE 390

TABLE 1. Baseline characteristics of cohorts

\begin{tabular}{|c|c|c|c|}
\hline Characteristic, $\mathrm{N}=99$ & Activities Outside the Home, $n=44$ & No Activities Outside the Home, $n=55$ & $\mathrm{p}$ Value \\
\hline \multicolumn{4}{|l|}{ Arthrodesis performed } \\
\hline 1 & $1(2.27)$ & $4(7.27)$ & 0.517 \\
\hline 2 & $2(4.55)$ & $2(3.64)$ & 1 \\
\hline 3 & $0(0.00)$ & $0(0.00)$ & 1 \\
\hline 4 & $0(0.00)$ & $0(0.00)$ & 1 \\
\hline
\end{tabular}

ASA = American Society of Anesthesiologists; GED = General Equivalency Diploma.

Unless otherwise indicated, values are expressed as the number of patients (\%). Boldface type indicates statistical significance.

not participate in activities outside the home $(n=43)$ experienced significant improvements in back pain, leg pain, disability, and quality of life at both 3 and 12 months postintervention (Table 4, Fig. 1).

\section{Multivariate Analysis}

A multivariate analysis was performed to determine

TABLE 2. Activity outside the home, complication rate, and minimally invasive versus open surgeries

\begin{tabular}{cccc}
\hline $\begin{array}{c}\text { Activities Outside } \\
\text { the Home }\end{array}$ & $\begin{array}{c}\text { Complication } \\
\text { Rate* }^{*}\end{array}$ & $\begin{array}{c}\text { Open } \\
\text { Approach }\end{array}$ & $\begin{array}{c}\text { Minimally Invasive } \\
\text { Approach }\end{array}$ \\
\hline Yes $(n=44)$ & $6(13.6 \%)$ & 38 & 6 \\
\hline No $(n=55)$ & $11(20.0 \%)$ & 43 & 12 \\
\hline
\end{tabular}

* Complications included incidental or unintended durotomy, new postoperative neurological deterioration, postoperative urinary retention or urinary tract infection, wound breakdown or dehiscence, and one episode of facet failure requiring reoperation. the independent association of activity outside the home and functional outcomes, both pre- and postintervention. Age, sex, smoking status, diabetes mellitus, depression, BMI, and patient activity outside the home were included $^{36}$ (Tables 5-7).

At baseline, patients with depression, a BMI of 25-29.9, a BMI of 30-34.9, and a BMI $\geq 35$ had worse back pain. Patients active outside the home reported comparatively less disability and higher quality of life (Table 5).

At 12 months postintervention, there were no statistically significant associations between age, sex, smoking, diabetes, depression, BMI, or being active outside the home with respect to back pain, leg pain, ODI score, or EQ-5D score ${ }^{36}$ (Table 6). However, patients who were active outside the home preoperatively had greater overall improvement in their reported disability than those who were not active outside the home at 12 months after decompressive surgery (Table 7).

\section{Discussion}

The present study represents an attempt at identifying

TABLE 3. Functional outcomes of cohorts at baseline, 3 months, and 12 months postintervention

\begin{tabular}{|c|c|c|c|c|c|}
\hline Outcome, $\mathrm{N}=99$ & Activities Outside the Home, $n=44$ & No Activities Outside the Home, $n=55$ & Mean Difference & $95 \% \mathrm{Cl}$ & p Value \\
\hline \multicolumn{6}{|l|}{ Back pain* } \\
\hline Baseline & $6.20 \pm 2.77$ & $7.40 \pm 2.03$ & 1.2 & 0.22 to 2.17 & $<0.001$ \\
\hline 3-mo & $2.06 \pm 2.03$ & $2.51 \pm 2.69$ & 0.45 & -0.62 to 1.52 & 0.437 \\
\hline 12-mo & $3.10 \pm 2.65$ & $2.74 \pm 3.00$ & 0.36 & -1.02 to 1.75 & 0.616 \\
\hline \multicolumn{6}{|l|}{ Leg pain* } \\
\hline Baseline & $6.55 \pm 2.71$ & $7.22 \pm 2.51$ & 0.67 & -0.37 to 1.71 & 0.209 \\
\hline 3-mo & $1.45 \pm 1.85$ & $3.26 \pm 3.31$ & 1.8 & 0.62 to 2.99 & $<0.001$ \\
\hline 12-mo & $2.52 \pm 2.55$ & $2.41 \pm 2.82$ & 0.11 & -1.22 to 1.43 & 0.88 \\
\hline \multicolumn{6}{|l|}{ Total EQ-5D score } \\
\hline Baseline & $0.62 \pm 0.20$ & $0.46 \pm 0.23$ & 0.16 & 0.07 to 0.24 & $<0.001$ \\
\hline 3-mo & $0.81 \pm 0.20$ & $0.74 \pm 0.24$ & 0.06 & -0.04 to 0.16 & 0.229 \\
\hline 12-mo & $0.80 \pm 0.14$ & $0.77 \pm 0.24$ & 0.03 & -0.07 to 0.12 & 0.59 \\
\hline \multicolumn{6}{|l|}{ Total ODI sum score } \\
\hline Baseline & $35.55 \pm 13.50$ & $51.35 \pm 14.14$ & 15.8 & 10.33 to 21.27 & $<0.001$ \\
\hline 3-mo & $14.18 \pm 11.58$ & $25.44 \pm 22.41$ & 11.26 & 3.48 to 19.04 & $<0.001$ \\
\hline 12-mo & $16.84 \pm 15.84$ & $20.71 \pm 19.07$ & 3.87 & -4.63 to 12.36 & 0.387 \\
\hline
\end{tabular}

Mean values are expressed \pm SD. Boldface type indicates statistical significance.

* On a scale of $0-10$ over the past 7 days. 
TABLE 4. Functional outcomes of cohorts at baseline, 3 months, and 12 months using paired testing

\begin{tabular}{crrrr}
\hline Functional Outcome, $N=76$ & Activities Outside the Home, $n=33$ & $p$ Value & No Activities Outside the Home, $n=43$ & $p$ Value \\
\hline Back pain* & & & & \\
\hline Baseline vs 3-mo & $4.53 \pm 3.28$ & $<0.001$ & $5.02 \pm 3.03$ & $<0.001$ \\
\hline Baseline vs 12-mo & $3.30 \pm 3.43$ & $<0.001$ & $4.50 \pm 3.29$ & $<0.001$ \\
\hline 3-mo vs 12-mo & $-1.24 \pm 3.25$ & 0.074 & $-0.37 \pm 2.39$ & 0.437 \\
\hline Leg pain* & & & & \\
\hline Baseline vs 3-mo & $5.35 \pm 2.88$ & $<0.001$ & $4.21 \pm 3.75$ & $<0.001$ \\
\hline Baseline vs 12-mo & $4.24 \pm 3.55$ & $<0.001$ & $4.85 \pm 3.22$ & $<.001$ \\
\hline 3-mo vs 12-mo & $-1.54 \pm 2.92$ & $<0.001$ & $0.78 \pm 2.35$ & 0.103 \\
\hline Total ODI sum score & & & & $<0.001$ \\
\hline Baseline vs 3-mo & $23.45 \pm 14.85$ & $<0.001$ & $26.00 \pm 17.68$ & $<0.001$ \\
\hline Baseline vs 12-mo & $18.97 \pm 13.49$ & $<0.001$ & $29.29 \pm 17.73$ & 0.861 \\
\hline 3-mo vs 12-mo & $-2.54 \pm 15.31$ & 0.415 & $0.52 \pm 14.92$ & $<0.001$ \\
\hline Total EQ-5D score & & & & $<0.001$ \\
\hline Baseline vs 3-mo & $-0.22 \pm 0.29$ & $<0.001$ & $-0.30 \pm 0.22$ & 0.645 \\
\hline Baseline vs 12-mo & $-0.21 \pm 0.21$ & $<0.001$ & $-0.27 \pm 0.24$ & $0.02 \pm 0.18$ \\
\hline 3-mo vs 12-mo & $0.04 \pm 0.16$ & 0.217 & & \\
\hline
\end{tabular}

Mean values are expressed \pm SD. Boldface type indicates statistical significance.

* On a scale of $0-10$ over the past 7 days.

TABLE 5. Baseline functional outcomes-multiple regression

\begin{tabular}{|c|c|c|c|c|}
\hline Variable & Back Pain & Leg Pain & ODI Score & $E Q-5 D$ \\
\hline \multicolumn{5}{|l|}{ Age, yrs } \\
\hline $40-49.9$ & $-0.194(1.370)$ & $-0.084(1.533)$ & $-4.439(8.252)$ & $0.163(0.121)$ \\
\hline $50-59.9$ & $-1.632(1.283)$ & $-1.093(1.436)$ & $-8.545(7.728)$ & $0.194(0.113)^{*}$ \\
\hline $60-69.9$ & $-0.409(1.288)$ & $-0.261(1.442)$ & $-5.506(7.762)$ & $0.255(0.113)^{* *}$ \\
\hline $70-80$ & $-1.520(1.308)$ & $0.468(1.465)$ & $-11.522(7.881)$ & $0.138(0.115)$ \\
\hline Male sex & $-0.540(0.609)$ & $-0.933(0.682)$ & $-1.195(3.667)$ & $-0.002(0.054)$ \\
\hline \multicolumn{5}{|l|}{ Smoker } \\
\hline Every day & $0.601(0.680)$ & $0.913(0.762)$ & $7.487(4.099)^{*}$ & $-0.104(0.060)^{*}$ \\
\hline Some days or occasionally & $-2.705(2.532)$ & $-0.197(2.835)$ & $-26.158(15.254)^{*}$ & $-0.033(0.223)$ \\
\hline \multicolumn{5}{|l|}{ Diabetes type 2} \\
\hline Insulin-dependent & $2.412(1.105)^{\star *}$ & $2.441(1.237)^{*}$ & $11.312(6.656)^{*}$ & $-0.266(0.097)^{\star * \star}$ \\
\hline Non-insulin-dependent & $1.179(0.910)$ & $1.339(1.019)$ & $4.313(5.484)$ & $-0.050(0.080)$ \\
\hline Depression disorder & $1.782(0.746)^{\star *}$ & $1.427(0.835)^{\star}$ & $3.502(4.494)$ & $-0.115(0.066)^{\star}$ \\
\hline \multicolumn{5}{|l|}{ BMI } \\
\hline $20-24.9$ & $2.382(1.468)$ & $-0.352(1.644)$ & $2.417(8.845)$ & $0.120(0.129)$ \\
\hline $25-29.9$ & $3.583(1.438)^{\star *}$ & $-0.454(1.610)$ & $9.771(8.666)$ & $0.147(0.127)$ \\
\hline $30-34.9$ & $3.847(1.555)^{\star *}$ & $0.464(1.741)$ & $12.102(9.367)$ & $0.024(0.137)$ \\
\hline$\geq 35$ & $4.448(1.586)^{* * *}$ & $0.409(1.776)$ & $16.021(9.555)^{*}$ & $0.037(0.140)$ \\
\hline Active outside the home & $-0.242(0.553)$ & $0.333(0.620)$ & $-11.759(3.334)^{* * *}$ & $0.127(0.049)^{* *}$ \\
\hline Intercept & $4.306(1.962)^{\star *}$ & $7.228(2.196)^{\star * *}$ & $45.747(11.818)^{* * *}$ & $0.250(0.173)$ \\
\hline Observations & 91 & 91 & 91 & 91 \\
\hline $\mathrm{R}^{2}$ & 0.314 & 0.195 & 0.428 & 0.378 \\
\hline Adjusted $\mathrm{R}^{2}$ & 0.177 & 0.034 & 0.313 & 0.254 \\
\hline Residual SE; df = 75 & 2.254 & 2.524 & 13.580 & 0.199 \\
\hline F statistic; $d f=15,75$ & $2.293^{* * *}$ & 1.211 & $3.735^{* * *}$ & $3.039 * * *$ \\
\hline
\end{tabular}

Values in parentheses represent the SE. Boldface type indicates statistical significance.

${ }^{*} p<0.1 ;{ }^{* *} p<0.05 ;{ }^{* * *} p<0.01$. 

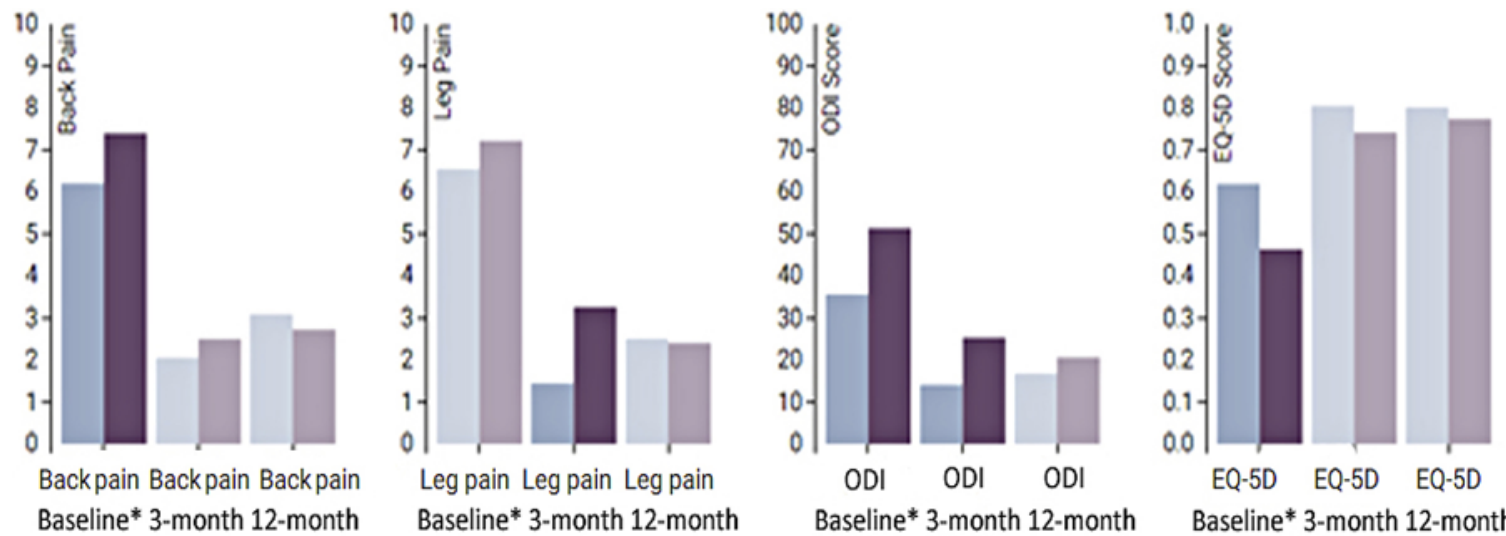

No activity outside of the home

Baseline* 3-month 12-month

FIG. 1. Bar graph representations of back pain, leg pain, ODI, and EQ-5D outcomes at baseline, 3-month, and 12-month time points for active versus nonactive cohorts. * The darker colors on the bar graph signify statistical significance between the two cohorts. Figure is available in color online only.

a preoperative factor associated with postoperative outcomes through retrospective review of a prospective database. Although this study design is an efficient way of providing valuable hypothesis-generating data, it remains subject to the limitations that can only be overcome by a randomized, prospective sample. ${ }^{6}$ Our study is novel in that it specifically focused on preoperative physical activ- ity outside the home and its relationship to surgical outcome following decompression surgery for symptomatic lumbar spinal stenosis.

Previous research with patient-reported outcomes has attempted to determine which patient characteristics may have a significant impact on postintervention outcomes. ${ }^{1,22}$ For some patient characteristics, such as the degree of

TABLE 6. One-year functional outcomes-multiple regression

\begin{tabular}{|c|c|c|c|c|}
\hline Variable & Back Pain & Leg Pain & ODI Score & $E Q-5 D$ \\
\hline \multicolumn{5}{|l|}{ Age, yrs } \\
\hline $40-49.9$ & $1.344(3.653)$ & $1.292(3.277)$ & $4.936(21.951)$ & $-0.110(0.262)$ \\
\hline $50-59.9$ & $0.359(3.502)$ & $-0.286(3.140)$ & $-2.778(20.691)$ & $0.127(0.251)$ \\
\hline $60-69.9$ & $0.555(3.501)$ & $1.046(3.147)$ & $5.053(20.957)$ & $-0.030(0.251)$ \\
\hline $70-80$ & $-0.367(3.569)$ & $-0.640(3.200)$ & $-2.198(21.319)$ & $-0.021(0.256)$ \\
\hline Male sex & $0.978(1.085)$ & $0.286(0.974)$ & $-0.927(6.591)$ & $-0.022(0.078)$ \\
\hline Smoker, every day & $-1.204(1.146)$ & $-0.842(1.031)$ & $4.114(6.959)$ & $0.001(0.082)$ \\
\hline \multicolumn{5}{|l|}{ Diabetes type 2} \\
\hline Insulin-dependent & $1.897(2.101)$ & $1.737(1.884)$ & $22.576(12.476)^{*}$ & $-0.189(0.151)$ \\
\hline Non-insulin-dependent & $1.200(1.543)$ & $1.617(1.388)$ & $-2.144(9.369)$ & $-0.117(0.111)$ \\
\hline Depression disorder & $2.499(1.378)^{*}$ & $1.965(1.236)$ & $13.719(8.372)$ & $-0.098(0.099)$ \\
\hline \multicolumn{5}{|l|}{ BMI } \\
\hline $20-24.9$ & $2.609(2.624)$ & $4.236(2.352)^{*}$ & $7.063(15.915)$ & $0.015(0.188)$ \\
\hline $25-29.9$ & $0.684(2.584)$ & $3.350(2.316)$ & 7.897 (15.668) & $-0.017(0.185)$ \\
\hline $30-34.9$ & $0.516(2.806)$ & $2.841(2.528)$ & $9.772(16.985)$ & $0.109(0.201)$ \\
\hline$\geq 35$ & $0.992(3.028)$ & $4.051(2.715)$ & $16.004(17.795)$ & $-0.079(0.217)$ \\
\hline Active outside the home & $0.776(0.958)$ & $0.685(0.874)$ & $1.728(5.806)$ & $-0.032(0.069)$ \\
\hline Intercept & $0.254(4.431)$ & $-2.022(3.977)$ & $5.195(26.421)$ & $0.840(0.318)^{* *}$ \\
\hline Observations & 58 & 57 & 59 & 58 \\
\hline $\mathrm{R}^{2}$ & 0.186 & 0.197 & 0.201 & 0.193 \\
\hline Adjusted $\mathrm{R}^{2}$ & -0.078 & -0.070 & -0.053 & -0.070 \\
\hline Residual SE & $3.069 ; \mathrm{df}=43$ & $2.752 ; \mathrm{df}=42$ & $18.642 ; \mathrm{df}=44$ & $0.220 ; \mathrm{df}=43$ \\
\hline F statistic & $0.704 ; \mathrm{df}=14,43$ & $0.738 ; d f=14,42$ & $0.792 ; d f=14,44$ & $0.735 ; d f=14,43$ \\
\hline
\end{tabular}

Values in parentheses represent the SE. Boldface type indicates statistical significance.

${ }^{*} p<0.1 ;{ }^{* *} p<0.05$. 
preoperative lumbar spinal canal stenosis, the results have been mixed. Whereas some investigators have suggested that more significant lumbar stenosis is associated with improved outcomes postoperatively, others have found little relationship between the area of stenosis and functional outcomes, pain, and postoperative ambulation status. ${ }^{1,31}$ Similarly, several studies have suggested that obese $(\mathrm{BMI}>30)$ patients are less likely to report improved outcomes with decompressive lumbar surgery than are nonobese patients. ${ }^{14,18}$ However, a more recent study suggests that whereas this may be true in the short term (3 months), there is no difference in outcomes between obese and nonobese patients at 1 year after lumbar surgery. ${ }^{9}$

Diabetes, smoking, depression, and medical comorbidities are more reliably associated with worse postoperative outcomes, whereas patients with high preoperative functional status have better postoperative outcomes. ${ }^{1-3,17,24,25,30,32}$ With respect to functional status, highly symptomatic patients have been shown to adopt a more sedentary lifestyle due to their symptoms. ${ }^{27}$

In this study, both patients who reported activity outside the home and those who were sedentary/inactive demonstrated improvement in their outcome scores at 3 and 12 months postintervention. However, patients who were not active outside their home reported greater back pain, worse disability, and lower quality of life preoperatively, and had a statistically significantly slower recovery in postoperative leg pain and disability at 3 months postintervention. These differences between the two cohorts disappeared at 1 year following surgery. This finding is similar to a previous study in which obese patients demonstrated slower initial improvement following surgical decompression for lumbar spinal stenosis, but ultimately reached outcomes equivalent to those of nonobese patients at 12 months postintervention. ${ }^{9}$ This suggests that patients with worse functional status at baseline take longer to recover after lumbar decompression, which may have value with respect to patient selection, preoperative counseling, and managing patient expectations when considering lumbar decompression surgery.

When examining the sedentary/inactive patient population, there was a statistically significant propensity for these patients to have diabetes and/or be smokers. The effects of these variables on lumbar spinal pathology have been well described. Smoking has been shown to lead to higher rates of disc degeneration and, in the event of lumbar fixation and fusion, decreased fusion success rates. ${ }^{15,30}$ Both variables have also shown association with factors that may affect patient satisfaction and happiness. Diabetes has been linked to higher rates of complications postoperatively, and smoking is associated with a greater likelihood of nursing home and rehabilitation center discharge disposition following surgery.,26

When adjusting for the covariates of diabetes and

TABLE 7. Difference between baseline and 1-year functional outcomes-multiple regression

\begin{tabular}{|c|c|c|c|c|}
\hline Variable & Back Pain & Leg Pain & ODI Score & $E Q-5 D$ \\
\hline \multicolumn{5}{|l|}{ Age, yrs } \\
\hline $40-49.9$ & $-1.736(4.011)$ & $1.625(4.269)$ & $-10.887(20.245)$ & $-0.409(0.264)$ \\
\hline $50-59.9$ & $-1.153(3.844)$ & $2.037(4.092)$ & $-5.446(19.083)$ & $-0.150(0.253)$ \\
\hline $60-69.9$ & $0.410(3.843)$ & $2.478(4.100)$ & $-4.503(19.329)$ & $-0.337(0.253)$ \\
\hline $70-80$ & $0.993(3.918)$ & $4.284(4.169)$ & $-4.149(19.663)$ & $-0.189(0.258)$ \\
\hline Male sex & $-1.614(1.191)$ & $-1.461(1.269)$ & $-2.803(6.079)$ & $0.038(0.078)$ \\
\hline Smoker, every day & $1.914(1.258)$ & $1.108(1.343)$ & $0.510(6.418)$ & $0.076(0.083)$ \\
\hline \multicolumn{5}{|l|}{ Diabetes type 2} \\
\hline Insulin-dependent & $-0.798(2.307)$ & $0.207(2.455)$ & $-4.057(11.506)$ & $0.093(0.152)$ \\
\hline Non-insulin-dependent & $-0.871(1.693)$ & $0.209(1.808)$ & $4.351(8.641)$ & $-0.092(0.111)$ \\
\hline Depression disorder & $-0.468(1.513)$ & $-0.053(1.611)$ & $-14.011(7.721)^{\star}$ & $0.007(0.100)$ \\
\hline \multicolumn{5}{|l|}{ BMI } \\
\hline $20-24.9$ & $-0.181(2.880)$ & $-4.550(3.065)$ & $-8.530(14.678)$ & $-0.202(0.190)$ \\
\hline $25-29.9$ & $3.217(2.836)$ & $-3.659(3.018)$ & $-4.989(14.450)$ & $-0.315(0.187)^{*}$ \\
\hline $30-34.9$ & $3.416(3.081)$ & $-2.572(3.293)$ & $-3.271(15.665)$ & $-0.076(0.203)$ \\
\hline$\geq 35$ & $4.947(3.324)$ & $-3.406(3.537)$ & $-5.564(16.412)$ & $-0.265(0.219)$ \\
\hline Active outside the home & $-0.910(1.052)$ & $-0.299(1.138)$ & $-12.180(5.355)^{\star *}$ & $-0.127(0.069)^{*}$ \\
\hline Intercept & $2.577(4.864)$ & $6.166(5.181)$ & $44.547(24.368)^{*}$ & $0.754(0.320)^{* *}$ \\
\hline Observations & 58 & 57 & 59 & 58 \\
\hline $\mathrm{R}^{2}$ & 0.303 & 0.188 & 0.225 & 0.342 \\
\hline Adjusted $\mathrm{R}^{2}$ & 0.076 & -0.082 & -0.022 & 0.128 \\
\hline Residual SE & $3.369 ; d f=43$ & $3.585, d f=42$ & $17.193, \mathrm{df}=44$ & $0.222, d f=43$ \\
\hline F statistic & $1.333 ; d f=14,43$ & $0.696 ; d f=14,42$ & $0.913 ; d f=14,44$ & $1.596 ; d f=14,43$ \\
\hline
\end{tabular}

Values in parentheses represent the SE. Boldface type indicates statistical significance.

${ }^{*} p<0.1 ;{ }^{* *} p<0.05$. 
smoking status in our multivariate analysis, activity level outside the home was the only variable to be associated with a statistically significant improvement in disability scores at 12 months postintervention. Interpretation of these results suggests that more active patients, those with activity levels above the activities of daily living, are less likely to suffer from disability from their lumbar spinal stenosis both preoperatively and after surgical intervention for their condition. Although patient functional status has been investigated previously, no study to date has specifically demonstrated an association with preoperative activity levels outside the home.,17

\section{Limitations of the Study}

There are several limitations inherent to this study. At 12 months postintervention, our follow-up data were at $76 \%$, which could be improved. Completeness of the data across time points will be addressed with future automated data extraction and abstraction. The observed power of the statistical analyses can be improved with larger sample sizes. This will occur as patient enrollment for the prospective observational registry continues and expands. Another inherent limitation is our inability to adjust for patient selection for surgery. Factors such as body habitus, comorbid diseases, and surgeon perception of an individual patient's likelihood of a successful outcome following surgery may not be adjusted for in this study. ${ }^{12,16,29}$ A longer-term study that might better capture late complications, morbidity, or mortality would probably offer more definitive information on patient-reported outcomes after lumbar spine surgery for stenosis. Finally, a more granular investigation of specific pathology such as the degree of stenosis, levels and location of lumbar stenosis, and specific surgical approaches used to treat it may help elucidate and delineate additional occult covariates.

\section{Conclusions}

Sedentary/inactive patients have a more protracted recovery after lumbar decompressive surgery for symptomatic lumbar spinal stenosis than do similar patients who are active preoperatively. However, sedentary/inactive patients can expect to reach similar long-term outcomes as patients who are active/perform activities outside the home preoperatively at 12 months after lumbar spine surgery.

\section{References}

1. Aalto TJ, Malmivaara A, Kovacs F, Herno A, Alen M, Salmi L, et al: Preoperative predictors for postoperative clinical outcome in lumbar spinal stenosis: systematic review. Spine (Phila Pa 1976) 31:E648-E663, 2006

2. Aalto T, Sinikallio S, Kröger H, Viinamäki H, Herno A, Leinonen V, et al: Preoperative predictors for good postoperative satisfaction and functional outcome in lumbar spinal stenosis surgery-a prospective observational study with a two-year follow-up. Scand J Surg 101:255-260, 2012

3. Arinzon Z, Adunsky A, Fidelman Z, Gepstein R: Outcomes of decompression surgery for lumbar spinal stenosis in elderly diabetic patients. Eur Spine J 13:32-37, 2004

4. Asher AL, Chotai S, Devin CJ, Speroff T, Harrell FE Jr, Nian H, et al: Inadequacy of 3-month Oswestry Disability Index outcome for assessing individual longer-term patient experience after lumbar spine surgery. J Neurosurg Spine 25:170-180, 2016

5. Asher AL, McCormick PC, Selden NR, Ghogawala Z, McGirt MJ: The National Neurosurgery Quality and Outcomes Database and NeuroPoint Alliance: rationale, development, and implementation. Neurosurg Focus 34(1):E2, 2013

6. Asher AL, Speroff T, Dittus RS, Parker SL, Davies JM, Selden N, et al: The National Neurosurgery Quality and Outcomes Database ( $\left.{ }^{2} \mathrm{QOD}\right)$ : a collaborative North American outcomes registry to advance value-based spine care. Spine (Phila Pa 1976) 39 (22 Suppl 1):S106-S116, 2014

7. Atlas SJ, Keller RB, Wu YA, Deyo RA, Singer DE: Long-term outcomes of surgical and nonsurgical management of lumbar spinal stenosis: 8 to 10 year results from the Maine Lumbar Spine Study. Spine (Phila Pa 1976) 30:936-943, 2005

8. Devlin NJ, Brooks R: EQ-5D and the EuroQol Group: past, present and future. Appl Health Econ Health Policy 15:127-137, 2017

9. Elsayed G, Davis MC, Dupépé EC, McClugage SG, Szerlip P, Walters BC, et al: Obese (BMI >30) patients have greater functional improvement and reach equivalent outcomes at 12 months following decompression surgery for symptomatic lumbar stenosis. World Neurosurg 105:884-894, 2017

10. EuroQol Group: EuroQol-a new facility for the measurement of health-related quality of life. Health Policy Amst Neth 16:199-208, 1990

11. Fairbank JC, Pynsent PB: The Oswestry Disability Index. Spine (Phila Pa 1976) 25:2940-2952, 2000

12. Forhan M, Salas XR: Inequities in healthcare: a review of bias and discrimination in obesity treatment. Can J Diabetes 37:205-209, 2013

13. Foulongne E, Derrey S, Ould Slimane M, Levèque S, Tobenas AC, Dujardin F, et al: Lumbar spinal stenosis: which predictive factors of favorable functional results after decompressive laminectomy? Neurochirurgie 59:23-29, 2013

14. Giannadakis C, Nerland US, Solheim O, Jakola AS, Gulati M, Weber C, et al: Does obesity affect outcomes after decompressive surgery for lumbar spinal stenosis? A multicenter, observational, registry-based study. World Neurosurg 84:1227-1234, 2015

15. Hadley MN, Reddy SV: Smoking and the human vertebral column: a review of the impact of cigarette use on vertebral bone metabolism and spinal fusion. Neurosurgery 41:116124, 1997

16. Kaminsky J, Gadaleta D: A study of discrimination within the medical community as viewed by obese patients. Obes Surg 12:14-18, 2002

17. Katz JN, Lipson SJ, Brick GW, Grobler LJ, Weinstein JN, Fossel AH, et al: Clinical correlates of patient satisfaction after laminectomy for degenerative lumbar spinal stenosis. Spine (Phila Pa 1976) 20:1155-1160, 1995

18. Knutsson B, Michaëlsson K, Sandén B: Obesity is associated with inferior results after surgery for lumbar spinal stenosis: a study of 2633 patients from the Swedish spine register. Spine (Phila Pa 1976) 38:435-441, 2013

19. Knutsson B, Sandén B, Sjödén G, Järvholm B, Michaëlsson $\mathrm{K}$ : Body mass index and risk for clinical lumbar spinal stenosis: a cohort study. Spine (Phila Pa 1976) 40:1451-1456, 2015

20. Marchand AA, Suitner M, O’Shaughnessy J, Châtillon CÉ, Cantin V, Descarreaux M: Effects of a prehabilitation program on patients' recovery following spinal stenosis surgery: study protocol for a randomized controlled trial. Trials 16:483, 2015

21. McGirt MJ, Parker SL, Hilibrand A, Mummaneni P, Glassman SD, Devin CJ, et al: Lumbar surgery in the elderly provides significant health benefit in the us health care system: patient-reported outcomes in 4370 patients from the $\mathrm{N}^{2} \mathrm{QOD}$ registry. Neurosurgery 77 (Suppl 4):S125-S135, 2015 
22. McGirt MJ, Sivaganesan A, Asher AL, Devin CJ: Prediction model for outcome after low-back surgery: individualized likelihood of complication, hospital readmission, return to work, and 12-month improvement in functional disability. Neurosurg Focus 39(6):E13, 2015

23. McGirt MJ, Speroff T, Dittus RS, Harrell FE Jr, Asher AL: The National Neurosurgery Quality and Outcomes Database $\left(\mathrm{N}^{2} \mathrm{QOD}\right)$ : general overview and pilot-year project description. Neurosurg Focus 34(1):E6, 2013

24. McKillop AB, Carroll LJ, Battié MC: Depression as a prognostic factor of lumbar spinal stenosis: a systematic review. Spine J 14:837-846, 2014

25. McKillop AB, Carroll LJ, Jones CA, Battié MC: The relation of social support and depression in patients with chronic low back pain. Disabil Rehabil 39:1482-1488, 2017

26. Murphy ME, Maloney PR, McCutcheon BA, Rinaldo L, Shepherd D, Kerezoudis P, et al: Predictors of discharge to a nonhome facility in patients undergoing lumbar decompression without fusion for degenerative spine disease. Neurosurgery 81:638-649, 2017

27. Norden J, Smuck M, Sinha A, Hu R, Tomkins-Lane C: Objective measurement of free-living physical activity (performance) in lumbar spinal stenosis: are physical activity guidelines being met? Spine J 17:26-33, 2017

28. Paulsen RT, Bouknaitir JB, Fruensgaard S, Carreron L, Andersen M: Patient are satisfied one year after decompression surgery for lumbar spinal stenosis. Dan Med J 63:63, 2016

29. Puhl RM, Andreyeva T, Brownell KD: Perceptions of weight discrimination: prevalence and comparison to race and gender discrimination in America. Int J Obes 32:992-1000, 2008

30. Sandén B, Försth P, Michaëlsson K: Smokers show less improvement than nonsmokers two years after surgery for lumbar spinal stenosis: a study of 4555 patients from the Swedish spine register. Spine (Phila Pa 1976) 36:1059-1064, 2011

31. Sigmundsson FG, Kang XP, Jönsson B, Strömqvist B: Correlation between disability and MRI findings in lumbar spinal stenosis: a prospective study of 109 patients operated on by decompression. Acta Orthop 82:204-210, 2011

32. Sinikallio S, Aalto T, Airaksinen O, Lehto SM, Kröger H, Viinamäki H: Depression is associated with a poorer outcome of lumbar spinal stenosis surgery: a two-year prospective follow-up study. Spine (Phila Pa 1976) 36:677-682, 2011

33. Turner JA, Ersek M, Herron L, Deyo R: Surgery for lumbar spinal stenosis. Attempted meta-analysis of the literature. Spine (Phila Pa 1976) 17:1-8, 1992

34. UAB Research: Is IRB review required for use of public datasets? (http://www.uab.edu/research/administration/offices/ IRB/FAQs/Pages/PublicDatasets.aspx) [Accessed March 20, 2018]

35. von Elm E, Altman DG, Egger M, Pocock SJ, Gøtzsche PC, Vandenbroucke JP: The Strengthening the Reporting of Observational Studies in Epidemiology (STROBE) statement: guidelines for reporting observational studies. Ann Intern Med 147:573-577, 2007

36. Weinstein JN, Tosteson TD, Lurie JD, Tosteson AN, Blood E, Hanscom B, et al: Surgical versus nonsurgical therapy for lumbar spinal stenosis. N Engl J Med 358:794-810, 2008

\section{Disclosures}

The authors report no conflict of interest concerning the materials or methods used in this study or the findings specified in this paper.

\section{Author Contributions}

Conception and design: Elsayed, Davis, Dupépé, McClugage, Walters, Hadley. Acquisition of data: Elsayed, Davis, Dupépé, McClugage, Szerlip. Analysis and interpretation of data: Erwood, Elsayed. Drafting the article: Erwood. Critically revising the article: Erwood, Elsayed, Walters, Hadley. Reviewed submitted version of manuscript: Erwood, Walters. Approved the final version of the manuscript on behalf of all authors: Erwood. Statistical analysis: Elsayed, Szerlip. Administrative/technical/material support: Davis, Dupépé, McClugage. Study supervision: Walters, Hadley.

\section{Correspondence}

Matthew S. Erwood: University of Alabama at Birmingham, AL. merwood@uabmc.edu. 